\title{
Spread through air spaces is a poor prognostic indicator in patients undergoing lobectomy for lung cancer: A systematic review and meta- analysis
}

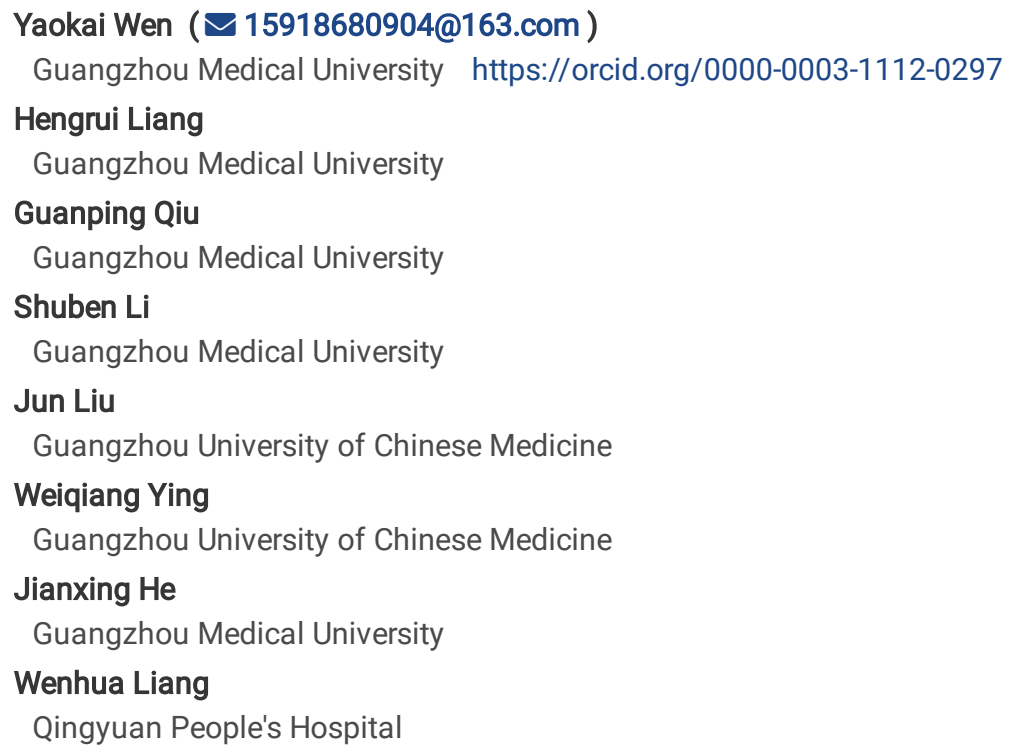

Research

Keywords: lung cancer, spread through air spaces, lobectomy, prognosis, meta-analysis

Posted Date: February 11th, 2020

DOI: https://doi.org/10.21203/rs.2.23116/v1

License: (c) (i) This work is licensed under a Creative Commons Attribution 4.0 International License. Read Full License 


\section{Abstract}

Background: Previous studies have confirmed the poor prognostic value of spread through air spaces (STAS) in patients undergoing limited resection for lung cancer. Nevertheless, its prognostic value remains controversial in patients undergoing lobectomy. Consequently, we aim to systematically and comprehensively evaluate the prognosis of patients with STAS undergoing lobectomy for lung cancer. Methods: An extensive search of literature databases was conducted. Recurrence-free survival (RFS) and overall survival (OS) were compared between patients with or without STAS undergoing lobectomy. In addition, results of the limited resection were also evaluated and presented. Results: 5 studies with 1531 patients reported the outcomes of lobectomy and 4 studies including 505 patients evaluated the survival of limited resection. In patients undergoing lobectomy, STAS was associated with significantly worse survival than non-STAS, including both RFS (HR=1.700; $95 \%$ Cl: $1.265-2.283 ; \mathrm{P} \otimes 0.001 ; \mathrm{P}$ for heterogeneity $=0.637 ; \mathrm{I}=0.0 \%)$ and $\mathrm{OS}(\mathrm{HR}=2.620 ; 95 \% \mathrm{Cl}: 1.138-6.031 ; \mathrm{P}=0.024 ; \mathrm{P}$ for heterogeneity $=0.128$; $12=51.4 \%$ ). STAS was also correlated with shorter RFS (HR=3.434, 95\% $\mathrm{Cl} 2.173$ to $5.428 ; \mathrm{P} \otimes 0.001 ; \mathrm{P}$ for heterogeneity $=0.828,12=0.0 \%$ ) and $0 \mathrm{~S}$ $(\mathrm{HR}=3.494,95 \% \mathrm{Cl} 2.128$ to $5.736 ; \mathrm{P} \otimes 0.001 ; \mathrm{P}$ for heterogeneity $=0.501,12=0.0 \%)$ in limited resection. Conclusions: STAS is a poor prognostic indicator in patients undergoing lobectomy for lung cancer.

\section{Background}

In the past, lung cancer invasion was established as (1) a histological subtype other than a lepidic pattern, (2) myofibroblastic stroma associated with invasive tumor cells, and (3) vascular or pleural invasion. In 2015, the concept of spread through air spaces (STAS) was proposed by World Health Organization (WHO) as a new pattern of invasion in adenocarcinoma on the basis of 2 validated large independent studies from the US ${ }^{1}$ and Germany ${ }^{2}$. It was defined as "micropapillary clusters, solid nests, or single cells spreading within air spaces beyond the edge of the main tumor" and regarded as the fourth category of lung cancer invasion. Though the concept of STAS was initially proposed for adenocarcinoma, current studies have confirmed that STAS was also observed in squamous cell carcinoma ${ }^{3-5}$, pleomorphic carcinoma ${ }^{6}$ and small-cell lung cancer ${ }^{7}$ and its prognostic value has aroused increasing attention in recent years.

Up till now, many previous studies have considered STAS as a poor prognostic indicator for both recurrence-free survival (RFS) and overall survival (OS). Initially, Warth A et al ${ }^{2}$ revealed that STAS related with shorter RFS and OS while Kadota $\mathrm{K}$ et al ${ }^{1}$ illustrated that STAS only predicted worse outcome for RFS in patients undergoing limited resection, implying that the prognostic value of STAS may vary under different surgical procedures. Since then, a variety of studies have been updated and the prognostic value of STAS in limited resection for lung cancer has been confirmed ${ }^{8-10}$. According to a previous meta-analysis ${ }^{11}$ calculating the hazard ratios (HRs) from multivariate analyses, the presence of STAS predicted both reduced RFS and OS in patients with non-small cell lung cancer (NSCLC), further validating its prognostic value in clinical practice. However, it is worth noting that most included studies in this meta-analysis consisted of multiple types of surgery and its subgroup analyses showed STAS in lobectomy was not related to reduced RFS while STAS in limited resection predicted significantly shorter RFS. Consequently, the overall conclusion of this meta-analysis could be influenced by the effect of the limited resection. In addition, in accordance to a recent study conducted by Yokoyama $S^{6}$, STAS in patients undergoing lobectomy for lung cancer predicted both shorter RFS and OS. Thus, in patients with lung cancer treated with lobectomy, it remains controversial whether STAS could result in worse prognosis and the prognostic value of STAS in lobectomy should be further evaluated with more current data.

Overall, the prognostic value of STAS in lobectomy remains uncertain. With more current data updated on lobectomy, it is the time to update a systematic review for a better recognition for its prognostic role in lobectomy for lung cancer.

\section{Methods}

Academic retrieval strategies:

An extensive literature retrieval of network databases including PubMed, Embase, Web of Science, Cochrane Library and Google Scholar was conducted to affirm all possibly relevant studies before April 2019. We amalgamated "lung cancer", "spread through air spaces", "STAS", "lobectomy", "limited resection", "sublobar resection", "segmentectomy", "wedge resection", "prognosis", "recurrence-free survival" and "overall survival" as well as their Medical Subject Headings (MeSH) terms. An additional manual search was carried out, containing the reference lists originating from retrieved review articles, primary studies, and abstracts from meetings.

All retrieved results were evaluated in accordance to the Preferred Reporting Items for Systematic Reviews and Meta-Analyses (PRISMA) statement. The original studies were screened gradually with the investigation on titles, abstracts, and full texts. The eligibility criteria were described as follows: (i) status of lung cancer and STAS should be confirmed through pathological examination. (ii) OS or RFS of patients with STAS and non-STAS was reported or could be estimated from the original article. (iii) all patients underwent complete surgical resection without neoadjuvant therapy. (v) articles were published or accepted in English with full texts and could be retrieved in the network databases mentioned above up to April 2019. Simultaneously, the exclusion criteria were stated as below: (i) hazard ratio (HR) and 95\% confidence 
interval (Cl) of OS or RFS could not be obtained directly or estimated from the original article. (ii) studies consisting of multiple types of surgical procedures while no separate outcomes on each type were reported (iii) patients underwent incomplete resection or received neoadjuvant chemotherapy. (iv) articles not written in English or couldn't be retrieved in the network databases mentioned above.

Data extraction and quality assessment:

4 investigators extracted the data independently (Y.K.W., G.P.Q., S.B.L. and H.R.L.), and disagreements among the four reviewers were resolved through discussion and eventually arrived at a consensus. The results were reviewed by the senior investigator (W.H.L.). All available information regarding target outcomes were acquired and included in a Microsoft Excel database. The basic data were recorded or estimated from each study: first author, year of publication, country, median age of patients, number of patients, incidence of STAS, histological type, pathological stage, surgical type, quality score. RFS or OS was obtained or estimated from each study to assess the prognostic value of STAS in lobectomy and limited resection.

Quality assessment of observational studies was carried out with the use of the Newcastle-Ottawa Scale, including 3 metrics: patient selection, comparability of groups, and the ascertainment of either the exposure or outcome of interest for case-control or cohort studies, respectively. The high-quality observational study was considered as a study with a quality score of $\geq 7$ stars with the total score of $0-9$ (allocated as stars).

Statistical analysis:

$\mathrm{HR}$ and $95 \% \mathrm{Cl}$ was obtained directly or estimated from the original article and they were used to evaluate for RFS and OS. HR $>1$ demonstrated a significantly worse prognosis for RFS and OS. Heterogeneity across studies was examined by the Cochran Q chi-square test and the $I^{2}$ statistic and statistical heterogeneity among studies was defined as $\mathrm{I}^{2}$ statistic greater than $50 \%$. A random-effects model was preferred if high heterogeneity of the studies $\left(P<0.1\right.$ or $\left.1^{2}>50 \%\right)$ was observed, and vice versa when there was no statistically significant heterogeneity. Publication bias was assessed using Funnel plots test, Begg's rank correlation test and Egger's linear regression method. Sensitivity analysis was performed by sequential removal of each study. The statistical analysis was performed in Stata software (version 12, StataCorp, TX). All the $P$ values were two tailed, and statistical significance was set as $P<0.05$.

\section{Results}

The Characteristics of the Studies and Quality Assessment:

A total of 176 studies were initially scanned from the previously mentioned 5 network databases before April, 2019. By inspecting the reference lists following retrieved articles and relevant reviewed articles, 10 studies were identified additionally. Of these, 144 studies were excluded through the removal of duplicated ones and identification in accordance to their titles and abstracts. After further confirmation in terms of full texts of the remaining 42 studies, 6 studies with a total of 2036 patients were finally included. Among them, 5 studies with 1531 patients reported the prognosis with regards to lobectomy and 4 studies including 505 patients evaluated the survival of limited resection. The retrieval process is elucidated in a PRISMA flow chart in Fig. 1.

All included studies were observational studies. As for the studies reporting the prognosis in lobectomy, they were performed in Japan $(n=3)$ and China $(n=2)$. Others reporting the results of limited resection were performed in Japan $(n=3)$ and China $(n=1)$. All studies were conducted after 2015. In addition, as far as the quality assessment is concerned, since all included studies were observation studies, the NewcastleOttawa Scale was applied and it manifested all included studies achieved high quality with a quality score of $\geq 7$ stars. The detailed characteristics and the quality assessment of each study were summarized in Table 1. 
Table 1

Demographics of the included studies

\begin{tabular}{|c|c|c|c|c|c|c|c|c|c|c|}
\hline Number & Study & Year & Country & $\begin{array}{l}\text { Median } \\
\text { age }\end{array}$ & $N(M / F)$ & STAS (+) & Type & Stage & $\begin{array}{l}\text { Surgical } \\
\text { type }\end{array}$ & $\begin{array}{l}\text { NOS } \\
\text { score }\end{array}$ \\
\hline 1 & $\begin{array}{l}\text { Kadota K } \\
a^{1}\end{array}$ & 2015 & Japan & 68 & $120(47 / 73)$ & $46(38.3 \%)$ & Adenocarcinoma & I & $\begin{array}{l}\text { Limited } \\
\text { resection }\end{array}$ & 7 \\
\hline 2 & $\begin{array}{l}\text { Kadota K } \\
\mathrm{b}^{1}\end{array}$ & 2015 & Japan & 68 & 291(117/174) & $109(37.5 \%)$ & Adenocarcinoma & I & Lobectomy & 7 \\
\hline 3 & $\begin{array}{l}\text { Shiono S } \\
a^{8}\end{array}$ & 2018 & Japan & 76 & $185(115 / 70)$ & $31(16.8 \%)$ & Mixed & $|A-||| A$ & $\begin{array}{l}\text { Limited } \\
\text { resection }\end{array}$ & 7 \\
\hline 4 & $\begin{array}{l}\text { Shiono S } \\
b^{8}\end{array}$ & 2018 & Japan & 68 & $329(170 / 159)$ & $73(22.2 \%)$ & Mixed & $\mid A-\| B$ & Lobectomy & 7 \\
\hline 5 & $\begin{array}{l}\text { Toyokawa } \\
\text { G D }^{9}\end{array}$ & 2018 & Japan & 71 & $82(40 / 42)$ & $31(37.8 \%)$ & Adenocarcinoma & $\mid A-\| A$ & $\begin{array}{l}\text { Limited } \\
\text { resection }\end{array}$ & 7 \\
\hline 6 & Yang $L^{14}$ & 2018 & China & NA & $242(111 / 131)$ & $81(33.47 \%)$ & Adenocarcinoma & IA1-IB & Lobectomy & 7 \\
\hline 7 & $\begin{array}{l}\text { Yokoyama } \\
\mathrm{S}^{6}\end{array}$ & 2018 & Japan & 62 & $35(27 / 8)$ & $14(40.0 \%)$ & $\begin{array}{l}\text { Pleomorphic } \\
\text { carcinoma }\end{array}$ & $\begin{array}{l}\text { IA2- } \\
\text { IIIB }\end{array}$ & Lobectomy & 8 \\
\hline 8 & Ren $Y a^{10}$ & 2019 & China & NA & 118(48/70) & $43(36.44 \%)$ & Adenocarcinoma & IA & $\begin{array}{l}\text { Sublobar } \\
\text { resection }\end{array}$ & 7 \\
\hline 9 & $\operatorname{Ren} Y b^{10}$ & 2019 & China & NA & $634(288 / 346)$ & $182(28.71 \%)$ & Adenocarcinoma & IA & Lobectomy & 7 \\
\hline
\end{tabular}

Table 2. Assessment for publication bias

\begin{tabular}{lcccc}
\hline & \multicolumn{4}{c}{ Assessment for publication bias } \\
\hline & RFS in lobectomy & OS in lobectomy & RFS in limited resection & OS in limited resection \\
\cline { 2 - 5 } Number of estimates & 5 & 3 & 4 & 3 \\
\hline P value for Begg's test & 0.221 & 1 & 0.734 & 0.296 \\
\hline P value for Egger's test & 0.184 & 0.327 & 0.205 & 0.382 \\
\hline
\end{tabular}

Relationship between STAS and prognosis in patients with lung cancer:

Prognosis of Patients with STAS undergoing lobectomy:

5 studies with 1531 patients reported RFS in the patients undergoing lobectomy. STAS occurred in 459 patients (30.0\%) while non-STAS occurred in 1072 patients (70.0\%). Our pooled analysis demonstrated among patients undergoing lobectomy for lung cancer, STAS was associated with shorter RFS compared with those without STAS(HR = 1.700; $95 \% \mathrm{Cl}: 1.265-2.283 ; \mathrm{P} \otimes 0.001 ; \mathrm{P}$ for heterogeneity $=0.637 ; \mathrm{I}^{2}=$ $0.0 \%$ ). In addition, OS were mentioned in 3 studies with 385 patients totally. We found that patients with STAS was correlated to shorter OS compared with those without STAS. (HR $=2.620,95 \% \mathrm{Cl} 1.138$ to $6.031 ; \mathrm{P}=0.024$; $\mathrm{P}$ for heterogeneity $=0.128, \mathrm{I}^{2}=51.4 \%$ ).

Prognosis of Patients with STAS undergoing limited resection:

4 studies including 505 patients provided the data concerning RFS in patients undergoing limited resection. Our result indicated that undergoing limited resection for lung cancer, patients with STAS was related with shorter RFS compared with those without STAS (HR $=3.434$, $95 \% \mathrm{Cl} 2.173$ to 5.428; $\mathrm{P} \otimes 0.001 ; \mathrm{P}$ for heterogeneity $=0.828, \mathrm{I}^{2}=0.0 \%$ ). Our pooled analysis of 3 studies with 911 patients also revealed that patients with STAS were linked to shorter OS compared with those without STAS (HR = 3.494, 95\% 2.128 to 5.736 ; P凶0.001; P for heterogeneity $\left.=0.501,1^{2}=0.0 \%\right)$.

Publication Bias and Sensitivity Analysis:

A sensitivity analysis was performed by removing each included study sequentially in each analysis. As for the RFS of both lobectomy and limited resection, our results indicated that none of the removal had changed the initial outcomes of analyses, indicating a valid stability of our outcomes. The sensitivity analysis elucidated that the OS of limited resection exhibited a validated result as well while the removal of the study 
by Yang $L$ et al has changed the initial outcome of OS in lobectomy. Also, a significant heterogeneity was observed regarding the outcome of OS in lobectomy ( $P$ for heterogeneity $=0.128, I^{2}=51.4 \%$ ). Consequently, this result should be explained carefully since it remains instable. The funnel plots indicated symmetric distributions at all results. In addition, in accordance to the results of both Begg's and Egger's tests, no publication bias was observed in all results.

\section{Discussion}

Our meta-analysis including 5 studies with 1531 patients demonstrated that STAS in lobectomy for lung cancer was associated with both worse RFS and OS. With more current data collected, we further confirmed the poor prognostic value of STAS in limited resection. To the best of our knowledge, this is the first meta-analysis with the latest data to assess the correlation between prognosis and STAS under lobectomy for lung cancer and the result confirmed its poor prognostic value under lobectomy for lung cancer.

Since the concept of STAS was proposed in 2015, various studies have been updated for investigating its prognostic value from different aspects. On one hand, some studies focused on its prognostic value under different predominant growth patterns of lung cancer. For example, Warth $A$ et $\mathrm{al}^{2}$ initially revealed that the impact of STAS on survival was specifically significant in papillary-predominant and micropapillarypredominant adenocarcinoma. Further confirmation was conducted by Morimoto $\mathrm{J}^{12}$ that STAS in micropapillary-predominant adenocarcinoma was significantly associated with lower 5-year RFS compared with those without STAS. Furthermore, they concluded that patients with both micropapillary-predominant adenocarcinoma and STAS had similar RFS compared with those with non-micropapillarypredominant adenocarcinoma in T stage 2. On the other hand, its prognostic value on different sizes of lung cancer was also evaluated. In accordance to Dai $C$ et a ${ }^{13}$, STAS could predict both reduced RFS and OS in adenocarcinoma $\leq 3 \mathrm{~cm}$ and $>2-3 \mathrm{~cm}$ while failed to predict survival in adenocarcinoma $\leq 2 \mathrm{~cm}$. Similar results were obtained from Yang $L$ et al ${ }^{14}$ that STAS could predict worse survival for patients with stage I adenocarcinomas $>2 \mathrm{~cm}$ while failed to predict survival for adenocarcinomas $\leq 2 \mathrm{~cm}$. In addition, studies ${ }^{4,15}$ concentrating on prognostic value of different extent and quantity of STAS were also conducted. In summary, based on the existing studies, we are supposed to attach great importance to its prognostic value.

Though STAS have been considered as a poor prognostic indicator for both RFS and OS by many previous studies mentioned above, its prognostic value under specific surgical procedure still remains uncertain since most of the existing studies consisted of multiple types of surgery. A meta-analysis conducted by Liu $\mathrm{H}^{11}$ has demonstrated that STAS was correlated with shorter RFS in patients undergoing limited resection while such correlation was not observed in patients with lobectomy. Based on their study, we add OR as another outcome indicator to comprehensively evaluate its prognostic value. According to our findings, STAS in limited resection for lung cancer was confirmed as a worse prognostic indicator for both RFS and OS. Furthermore, with more current data collected, our study denoted that STAS was associated with significantly reduced RFS in patients undergoing lobectomy for lung cancer, which is different from previous studies. Since Kadota $\mathrm{K}_{\text {et al }}{ }^{1}$ initially revealed that STAS in lobectomy for lung cancer was not related to either any or distant recurrence, the debate on its prognostic value in lobectomy has never stopped. Following studies $8,10,14$ also illustrated similar results. It is worth noting that their results exhibited trends towards to worse RFS though without statistical significance. Nevertheless, Yokoyama $\mathrm{S}$ et al ${ }^{6}$ indicated that STAS in lobectomy for lung cancer could predict both significantly worse RFS and OS for the first time though with a limited number of patients. Consequently, with more data collected concerning STAS in lobectomy compared with Liu $\mathrm{H}$ et al ${ }^{11}$, we conducted this updated meta-analysis to further assess its prognostic value in lobectomy for lung cancer. To the best of our knowledge, this is the first meta-analysis confirming the poor prognostic value of STAS in lobectomy for lung cancer. In addition, though high heterogeneity and instability were observed in our result regarding the OS in patients undergoing lobectomy, we assessed the OS of patients with STAS undergoing lobectomy for lung cancer for the first time and the result suggested that STAS could lead to worse OS in patients undergoing lobectomy for lung cancer. Therefore, based on the results of both worse RFS and OS, we are supposed to take the prognostic value of STAS very seriously for patients undergoing lobectomy for lung cancer in future clinical practice.

Meanwhile, several limitations in our study could not be neglected. First, our studies consisted of retrospective nonrandomized comparisons, increasing the risk of potential selection and reporting bias. Second, as mentioned above, our result of OS in patients with STAS undergoing lobectomy for lung cancer showed high heterogeneity and instability, which should be explained very carefully. After the removal of the study by Yang $L$ et al, the initial outcome was changed and no correlation was found between STAS and OS in patients undergoing lobectomy for lung cancer. After carefully assessing their clinicopathological characteristics, we found predominant subtypes in composition varied across different studies. According to Ren $\mathrm{Y}$ et al, lepidic pattern accounted for $11 \%$ of patients with STAS undergoing lobectomy and $43 \%$ of patients without STAS. Meanwhile, in the study conducted by Yang $L$ et al, lepidic subtype accounted for $1.23 \%$ of patients with STAS undergoing lobectomy and $13.7 \%$ of patients without STAS. It is widely acknowledged that lepidic adenocarcinoma is a kind of invasive non-mucinous adenocarcinoma associated with favorable prognosis compared with other subtypes for its lower invasiveness ${ }^{16-20}$. Therefore, our conclusion on worse OS for lobectomy could be affected by the different ratio of predominant subtypes of lung cancer with different invasiveness in different study and our conclusion should be further assessed in the condition of well-matched predominant subtypes.

Page 5/12 
Though our study has confirmed that STAS was associated with worse survival, the underlying mechanism remains unknown. Consequently, with the updated studies reporting its prognostic value in clinical practice, it is also the time for us to explore its mechanisms leading to worse survival. Wang $S$ et $\mathrm{al}^{21}$ have confirmed that STAS was associated with multiple aggressive characteristics. Among them, pleural invasion and vascular invasion have been defined as kinds of lung cancer invasion ${ }^{22,23}$. Thus, the worse survival of patients with STAS could be a kind of reflection of the aggressive characteristics mentioned above and the mechanism between STAS and relevant aggressive characteristics are also worth exploring. In addition, though many previous have elucidated that lobectomy was associated with better outcome for survival compared with limited resection in patients with STAS, limited resection was also believed to offer better pulmonary function in early stage lung cancer $^{24-33}$. Thus, it is essential for thoracic surgeons to select the appropriate surgical procedure depending on the status of STAS of patients during the operation. However, no clinicopathological standards have been established to identify STAS in frozen sections and some previous studies ${ }^{34}$ suggested that frozen sections could not effectively identify STAS, which might influence surgeons' decisions during the surgery and result in worse prognosis for patients. Thus, a well-established standard of STAS for frozen sections will be urgently needed in the future. Furthermore, the role of STAS in postoperative treatments is still not clear. Future large-scales studies should find out whether we should use postoperative treatments in patients with STAS and which type should be the optimal one.

\section{Conclusion}

The presence of STAS is a poor prognostic indicator in patients undergoing lobectomy for lung cancer. Future clinical practice should attach great importance to its prognostic value, independent of the surgical procedure.

\section{Declarations}

\section{Ethics approval and consent to participate:}

Not applicable

\section{Consent for publication:}

Not applicable

\section{Availability of data and materials:}

The datasets used and/or analysed during the current study are available from the corresponding author on reasonable request

\section{Competing interests:}

The authors declare that they have no competing interests

\section{Funding:}

This work was supported by the grant 2016YFC0905400 from the National Key R\&D Program of China; China National Science Foundation (Grant No. 81871893 \& No. 81501996); Key Project of Guangzhou Scientific Research Project (Grant No. 201804020030); High-level university construction project of Guangzhou medical university (Grant No. 20182737, 201721007, 201715907, 2017160107); IVATS National key R \& D Program (Grant No. 2017YFC0907903 \& 2017YFC0112704)

\section{Authors' contributions:}

Study conception and design was contributed by Y.W., H.L. and G.Q.; acquisition of data was performed by Y.W., H.L., G.Q., S.L. and W.L.; interpretation of data was done by Y.W., H.L., G.Q., S.L. and W.L.; Y.W., H.L., G.Q., J.L., W.Y. and J.H. drafted the article; critical revision was carried out by Y.W., H.L., G.Q., W.L., S.L., J.L., W.Y. and J.H.; final approval was done by Y.W., H.L., G.Q., W.L., S.L., J.L., W.Y. and J.H.; agreement to be accountable for all aspects of work was provided by Y.W., H.L., G.Q., W.L., S.L., J.L., W.Y. and J.H.

\section{Acknowledgements:}

Not applicable

\section{References}

1. Kadota K, Nitadori J, Sima CS, et al. Tumor Spread through Air Spaces is an Important Pattern of Invasion and Impacts the Frequency and Location of Recurrences after Limited Resection for Small Stage I Lung Adenocarcinomas. J Thorac Oncol. May 2015;10(5):806-814. 
2. Warth A, Muley T, Kossakowski CA, et al. Prognostic Impact of Intra-alveolar Tumor Spread in Pulmonary Adenocarcinoma. The American journal of surgical pathology. Jun 2015;39(6):793-801.

3. Kadota K, Kushida Y, Katsuki N, et al. Tumor Spread Through Air Spaces Is an Independent Predictor of Recurrence-free Survival in Patients With Resected Lung Squamous Cell Carcinoma. The American journal of surgical pathology. Aug 2017;41(8):1077-1086.

4. Lu S, Tan KS, Kadota K, et al. Spread through Air Spaces (STAS) Is an Independent Predictor of Recurrence and Lung Cancer-Specific Death in Squamous Cell Carcinoma. J Thorac Oncol. Feb 2017;12(2):223-234.

5. Yanagawa N, Shiono S, Endo M, Ogata SY. Tumor spread through air spaces is a useful predictor of recurrence and prognosis in stage I lung squamous cell carcinoma, but not in stage II and III. Lung Cancer. Jun 2018;120:14-21.

6. Yokoyama S, Murakami T, Tao H, et al. Tumor Spread Through Air Spaces Identifies a Distinct Subgroup With Poor Prognosis in Surgically Resected Lung Pleomorphic Carcinoma. Chest. Oct 2018;154(4):838-847.

7. Toyokawa G, Yamada Y, Tagawa T, et al. High Frequency of Spread Through Air Spaces in Resected Small Cell Lung Cancer. Anticancer Res. Mar 2018;38(3):1821-1825.

8. Shiono S, Endo M, Suzuki K, Yarimizu K, Hayasaka K, Yanagawa N. Spread Through Air Spaces Is a Prognostic Factor in Sublobar Resection of Non-Small Cell Lung Cancer. Ann Thorac Surg. Aug 2018;106(2):354-360.

9. Toyokawa G, Yamada Y, Tagawa T, Oda Y. Significance of spread through air spaces in early-stage lung adenocarcinomas undergoing limited resection. Thorac Cancer. Oct 2018;9(10):1255-1261.

10. Ren Y, Xie H, Dai C, et al. Prognostic Impact of Tumor Spread Through Air Spaces in Sublobar Resection for 1A Lung Adenocarcinoma Patients. Ann Surg Oncol. Mar 182019.

11. Liu H, Yin Q, Yang G, Qie P. Prognostic Impact of Tumor Spread Through Air Spaces in Non-small Cell Lung Cancers: a Meta-Analysis Including 3564 Patients. Pathol Oncol Res. Feb 142019.

12. Morimoto J, Nakajima T, Suzuki H, et al. Impact of free tumor clusters on prognosis after resection of pulmonary adenocarcinoma. $J$ Thorac Cardiovasc Surg. Jul 2016;152(1):64-72 e61.

13. Dai C, Xie H, Su H, et al. Tumor Spread through Air Spaces Affects the Recurrence and Overall Survival in Patients with Lung Adenocarcinoma >2 to $3 \mathrm{~cm}$. J Thorac Oncol. Jul 2017;12(7):1052-1060.

14. Yang $L$, Yang Y, Ma P, et al. Spread through air spaces predicts a worse survival in patients with stage I adenocarcinomas $>2 \mathrm{~cm}$ after radical lobectomy. J Thorac Dis. Sep 2018;10(9):5308-5317.

15. Uruga H, Fujii T, Fujimori S, Kohno T, Kishi K. Semiquantitative Assessment of Tumor Spread through Air Spaces (STAS) in Early-Stage Lung Adenocarcinomas. J Thorac Oncol. Jul 2017;12(7):1046-1051.

16. Borczuk AC. Prognostic considerations of the new World Health Organization classification of lung adenocarcinoma. Eur Respir Rev. Dec 2016;25(142):364-371.

17. Yoshizawa A, Sumiyoshi S, Sonobe M, et al. Validation of the IASLC/ATS/ERS lung adenocarcinoma classification for prognosis and association with EGFR and KRAS gene mutations: analysis of 440 Japanese patients. J Thorac Oncol. Jan 2013;8(1):52-61.

18. Russell PA, Wainer Z, Wright GM, Daniels M, Conron M, Williams RA. Does lung adenocarcinoma subtype predict patient survival?: A clinicopathologic study based on the new International Association for the Study of Lung Cancer/American Thoracic Society/European Respiratory Society international multidisciplinary lung adenocarcinoma classification. J Thorac Oncol. Sep 2011;6(9):1496-1504.

19. Murakami S, Ito H, Tsubokawa N, et al. Prognostic value of the new IASLC/ATS/ERS classification of clinical stage IA lung adenocarcinoma. Lung Cancer. Nov 2015;90(2):199-204.

20. Warth A, Muley T, Meister M, et al. The novel histologic International Association for the Study of Lung Cancer/American Thoracic Society/European Respiratory Society classification system of lung adenocarcinoma is a stage-independent predictor of survival. Journal of clinical oncology : official journal of the American Society of Clinical Oncology. May 1 2012;30(13):1438-1446.

21. Wang S, Hao J, Qian C, Wang H. Tumor Spread Through Air Spaces Is a Survival Predictor in Non-Small-Cell Lung Cancer. Clin Lung Cancer. May 292019.

22. Warth A, Beasley MB, Mino-Kenudson M. Breaking New Ground: The Evolving Concept of Spread through Air Spaces (STAS). J Thorac Oncol. Feb 2017;12(2):176-178.

23. Warth A. Spread through air spaces (STAS): a comprehensive update. Transl Lung Cancer Res. Oct 2017;6(5):501-507.

24. Martinucar A, Nakas A, Pilling J, West K, Waller D. A case-matched study of anatomical segmentectomy versus lobectomy for stage I lung cancer in high-risk patients. European Journal of Cardio-Thoracic Surgery. 2005;27(4):675-679.

25. Kim SJ, Lee YJ, Park JS, et al. Changes in pulmonary function in lung cancer patients after video-assisted thoracic surgery. The Annals of thoracic surgery. Jan 2015;99(1):210-217. 
26. Watanabe A, Ohori S, Nakashima S, et al. Feasibility of video-assisted thoracoscopic surgery segmentectomy for selected peripheral lung carcinomas. European journal of cardio-thoracic surgery : official journal of the European Association for Cardio-thoracic Surgery. May 2009;35(5):775-780; discussion 780.

27. Harada H, Okada M, Sakamoto T, Matsuoka H, Tsubota N. Functional advantage after radical segmentectomy versus lobectomy for lung cancer. The Annals of thoracic surgery. Dec 2005;80(6):2041-2045.

28. Koike T, Koike T, Sato S, et al. Lobectomy and limited resection in small-sized peripheral non-small cell lung cancer. Journal of thoracic disease. Nov 2016;8(11):3265-3274.

29. Kobayashi N, Kobayashi K, Kikuchi S, et al. Long-term pulmonary function after surgery for lung cancer. Interactive cardiovascular and thoracic surgery. May 1 2017;24(5):727-732.

30. Macke RA, Schuchert MJ, Odell DD, Wilson DO, Luketich JD, Landreneau RJ. Parenchymal preserving anatomic resections result in less pulmonary function loss in patients with Stage I non-small cell lung cancer. Journal of cardiothoracic surgery. Apr 1 2015;10:49.

31. Saito H, Nakagawa T, Ito M, Imai K, Ono T, Minamiya Y. Pulmonary Function After Lobectomy Versus Segmentectomy in Patients with Stage I Non-Small Cell Lung Cancer. World journal of surgery. 2014;38(8):2025-2031.

32. Yoshimoto K, Nomori H, Mori T, et al. Quantification of the impact of segmentectomy on pulmonary function by perfusion single-photonemission computed tomography and multidetector computed tomography. The Journal of thoracic and cardiovascular surgery. May 2009;137(5):1200-1205.

33. Keenan RJ, Landreneau RJ, Maley RH, et al. Segmental resection spares pulmonary function in patients with stage I lung cancer. The Annals of thoracic surgery. 2004;78(1):228-233.

34. Walts AE, Marchevsky AM. Current Evidence Does Not Warrant Frozen Section Evaluation for the Presence of Tumor Spread Through Alveolar Spaces. Arch Pathol Lab Med. Jan 2018;142(1):59-63.

\section{Figures}



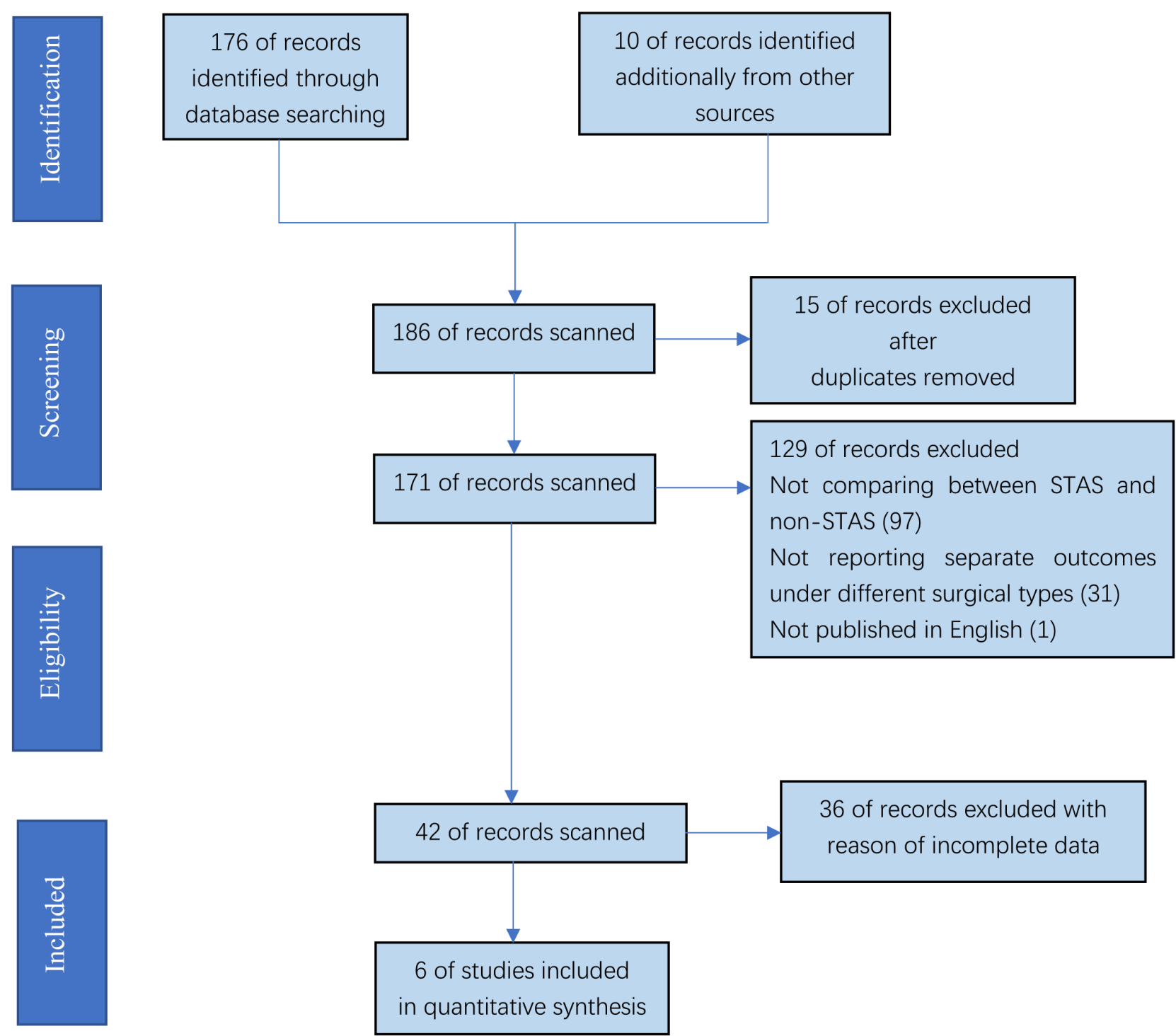

\section{Figure 1}

PRISMA flow diagram including the search strategy and identification of studies used in meta-analysis.

A

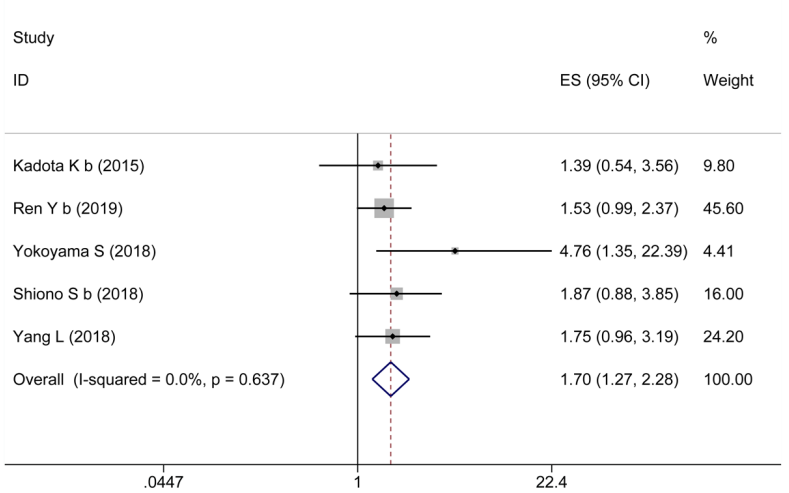

B

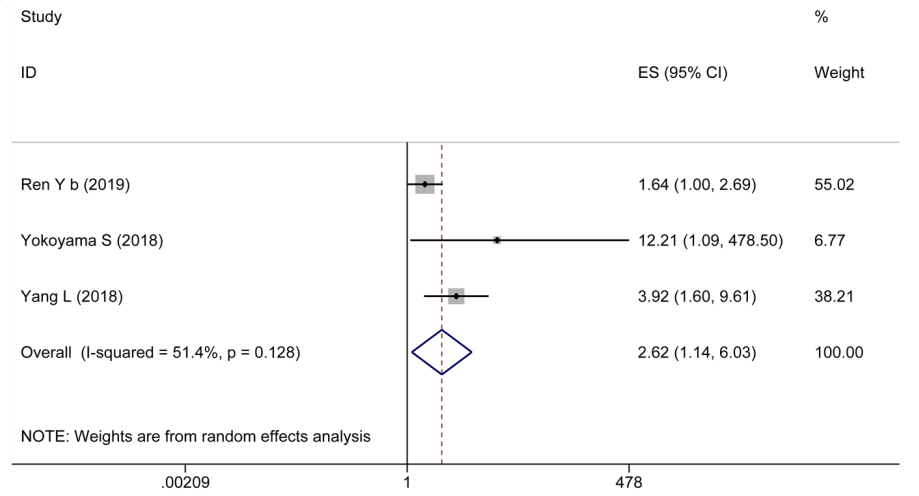

Figure 2. Outcomes of the association between STAS and RFS (A) or OS rate (B) in lobectomy for lung cancer 

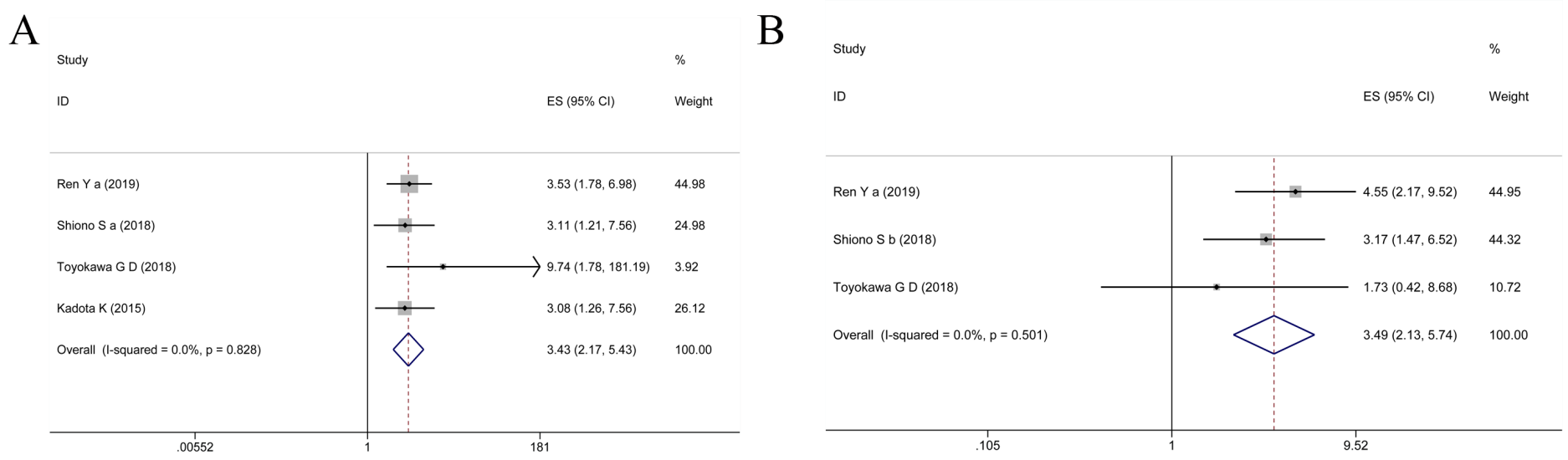

Figure 3. Outcomes of the association between STAS and RFS (A) or OS rate (B) in limited resection for lung cancer

Figure 3

Outcomes of the association between STAS and RFS (A) or OS rate (B) in limited resection for lung cancer
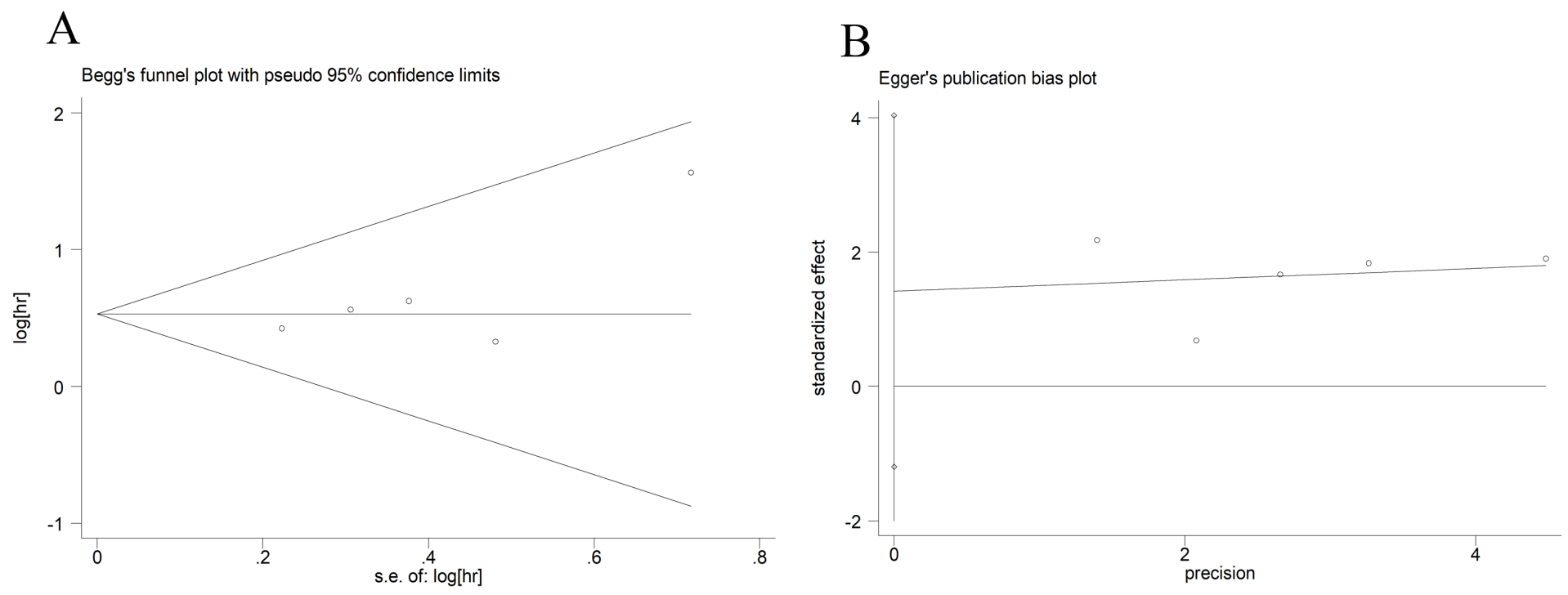

Figure 4. Publication bias for RFS of patients undergoing lobectomy for lung cancer (A) Begg's funnel plot (B) Egger's publication bias plot

\section{Figure 4}

Publication bias for RFS of patients undergoing lobectomy for lung cancer (A) Begg's funnel plot (B) Egger's publication bias plot 
A

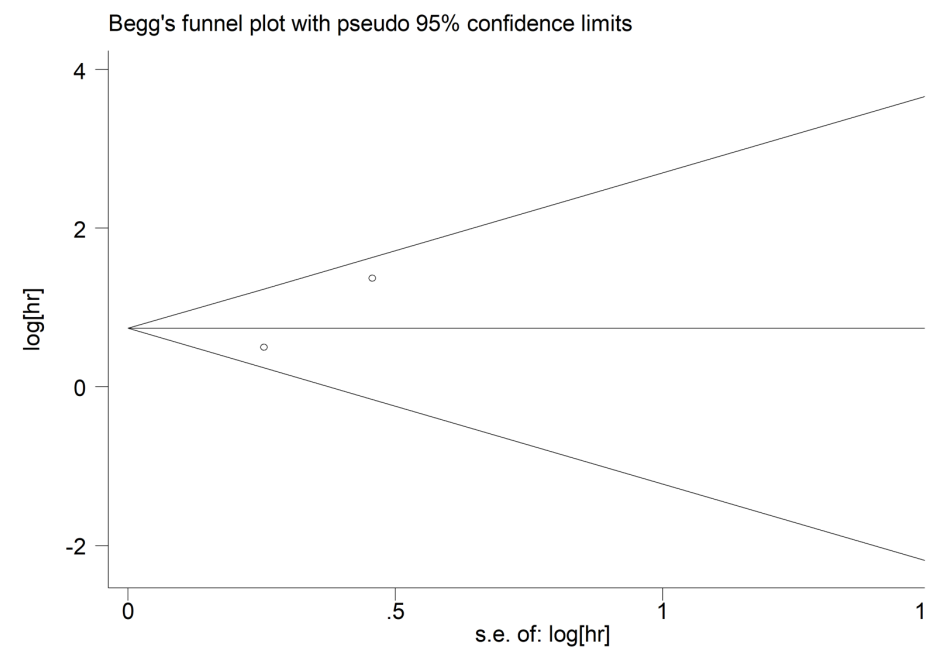

\section{B}

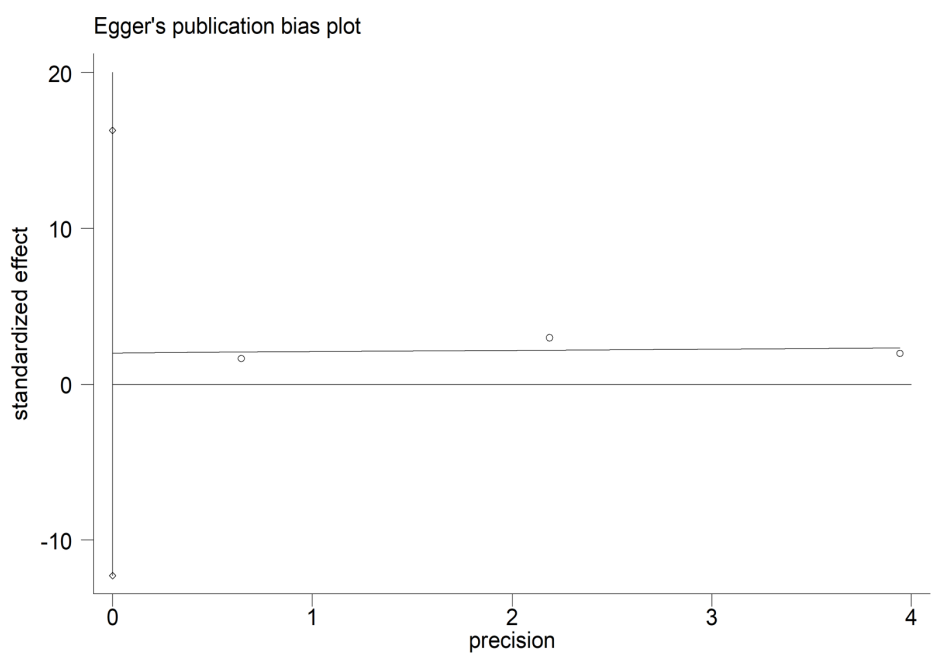

Figure 5. Publication bias for OS of patients undergoing lobectomy for lung cancer (A) Begg's funnel plot (B) Egger's publication bias plot

\section{Figure 5}

Publication bias for OS of patients undergoing lobectomy for lung cancer (A) Begg's funnel plot (B) Egger's publication bias plot
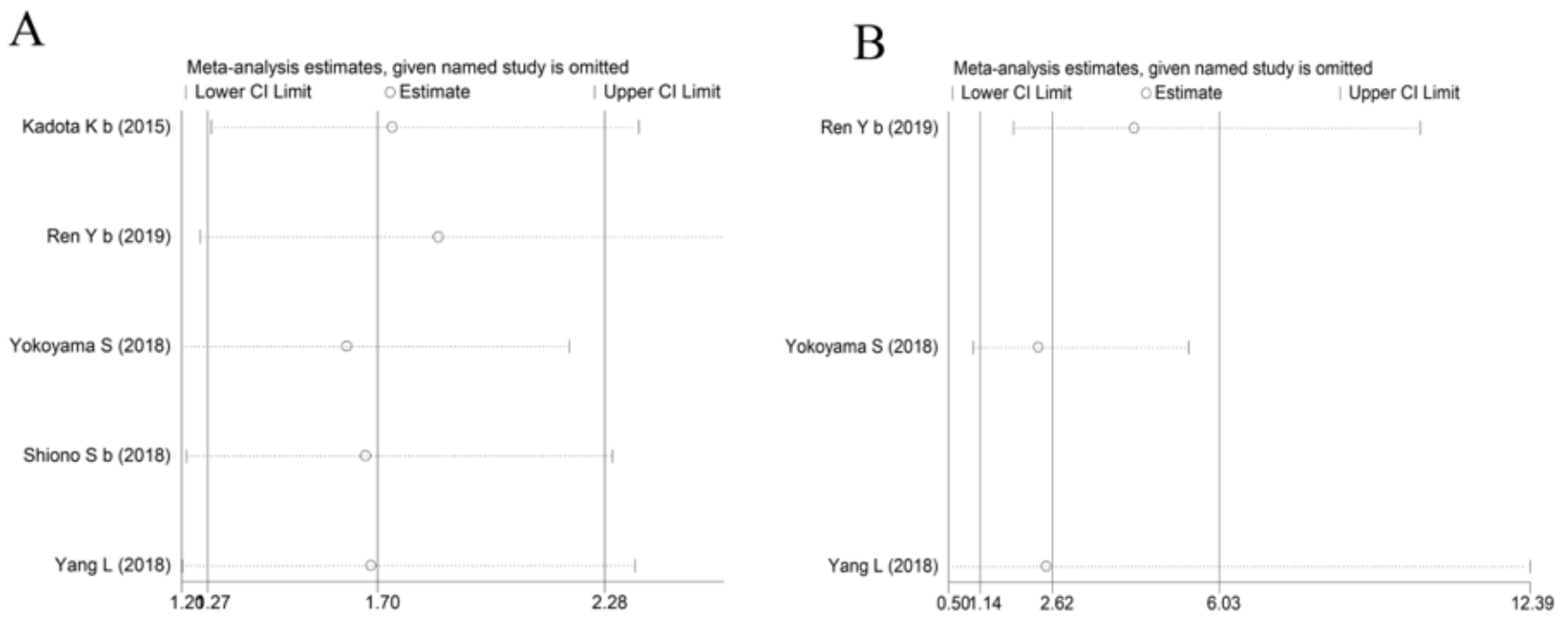

Figure 6. Sensitivity analyses for patients undergoing lobectomy for lung cancer (A) Sensitivity analysis for RFS (B) Sensitivity analysis for OS

\section{Figure 6}

Sensitivity analyses for patients undergoing lobectomy for lung cancer (A) Sensitivity analysis for RFS (B) Sensitivity analysis for OS 
A

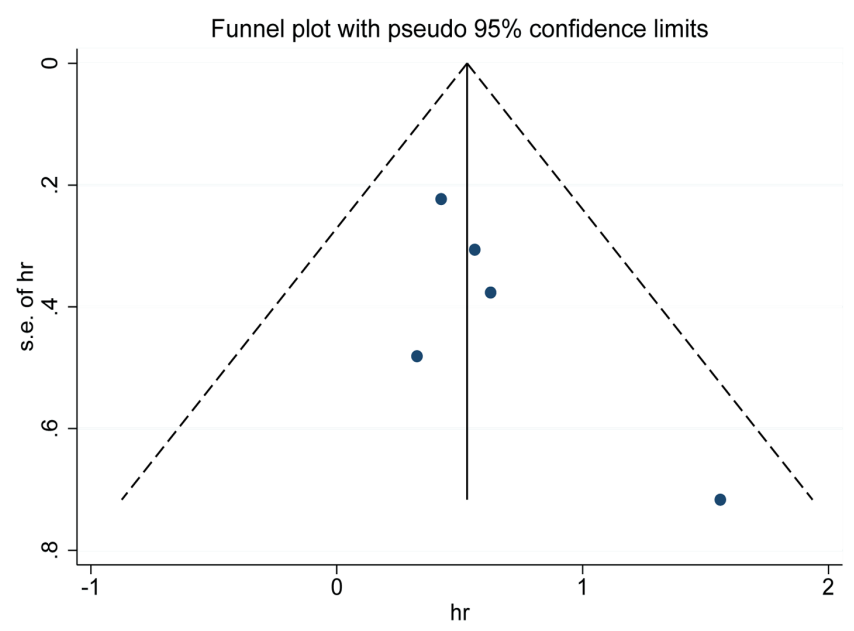

B

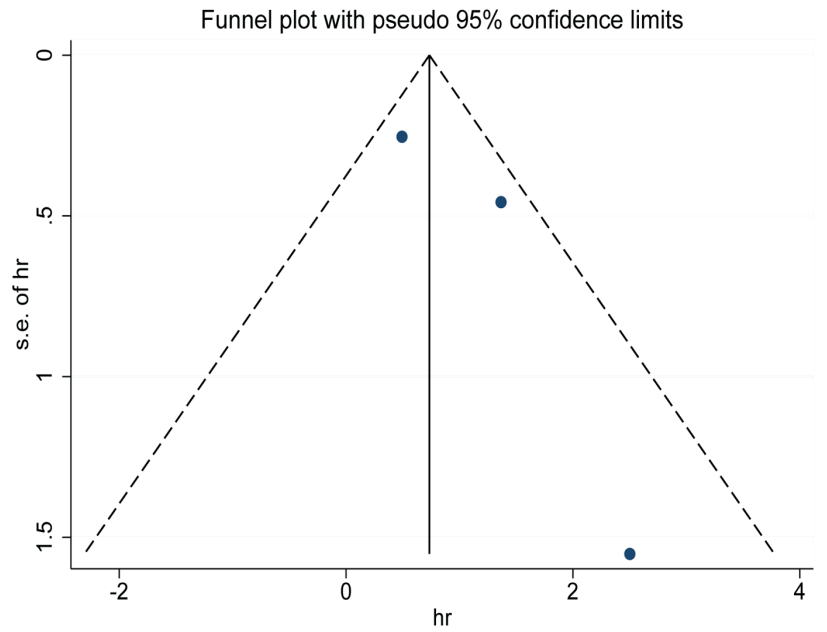

Figure 7. Funnel plots for patients undergoing lobectomy for lung cancer (A) Funnel plot for RFS (B) Funnel plot for OS

\section{Figure 7}

Funnel plots for patients undergoing lobectomy for lung cancer (A) Funnel plot for RFS (B) Funnel plot for OS 